\title{
Cortical Thickness in Congenital Amusia: When Less Is Better Than More
}

\author{
Krista L. Hyde, ${ }^{1,2}$ Jason P. Lerch, ${ }^{3}$ Robert J. Zatorre, ${ }^{1}$ Timothy D. Griffiths, ${ }^{4}$ Alan C. Evans, ${ }^{1}$ and Isabelle Peretz ${ }^{2}$ \\ ${ }^{1}$ Montreal Neurological Institute, McGill University, Montreal, Quebec, Canada H3A 2B4, ${ }^{2}$ Department of Psychology, University of Montreal, Montreal, \\ Quebec, Canada H3C 3J7, ${ }^{3}$ Hospital for Sick Children, Toronto, Ontario, Canada M5G 1X8, and ${ }^{4}$ Newcastle University Medical School, Newcastle upon Tyne \\ NE2 4HH, United Kingdom
}

Congenital amusia (or tone deafness) is a lifelong disorder characterized by impairments in the perception and production of music. A previous voxel-based morphometry (VBM) study revealed that amusic individuals had reduced white matter in the right inferior frontal gyrus (IFG) relative to musically intact controls (Hyde et al., 2006). However, this VBM study also revealed associated increases in gray matter in the same right IFG region of amusics. The objective of the present study was to better understand this morphological brain anomaly by way of cortical thickness measures that provide a more specific measure of cortical morphology relative to VBM. We found that amusic subjects $(n=21)$ have thicker cortex in the right IFG and the right auditory cortex relative to musically intact controls $(n=$ 26). These cortical thickness differences suggest the presence of cortical malformations in the amusic brain, such as abnormal neuronal migration, that may have compromised the normal development of a right frontotemporal pathway.

Key words: congenital amusia; tone deafness; music; brain; cortical thickness; MRI

\section{Introduction}

About four percent of the population is not able to share in the universal enjoyment and production of music (Kalmus and Fry, 1980). Such individuals are afflicted with congenital amusia, a lifelong disability that prevents otherwise normal functioning individuals from developing basic musical skills. The condition has been variously termed note deafness, tone deafness, tune deafness, and, more recently, congenital amusia (Ayotte et al., 2002). These terms refer to the same condition in which adults who report lifelong difficulties with music, exhibit a deficit in detecting pitch changes in melodies. The term amusia seems preferable to acknowledge the possibility that there may exist as many forms of congenital amusias as in acquired amusias that occur consequent to accidental brain damage. All congenital amusia cases reported to date have failed to acquire normal music perception and music recognition abilities, despite normal neurological history, hearing, language, intelligence and memory. The condition appears to be music specific because amusic individuals have a normal understanding of speech and prosody. However, in contrast to musically intact controls, amusic persons are unable to

\footnotetext{
Received March 27, 2007; revised Sept. 13, 2007; accepted 0ct. 22, 2007.

This work was supported by funds from Fonds de Recherche en Santé du Québec and Centre for Interdisciplinary Research in Music Media and Technology to K.L.H., Canadian Institutes of Health Research to I.P. and R.Z., and the Wellcome Trust (United Kingdom) to T.G. We thank Elizabeth Coulthard and Philip English for collecting the Newcastle MRI data, and Jessica Foxton for collecting the Newcastle behavioral data. We thank Marc Bouffard and the MRI technicians of the McConnell Brain Imaging Centre of the Montreal Neurological Institute for technical assistance in the acquisition of the Montreal MRI data. We thank our subjects for their continued cooperation.

Correspondence should be addressed to Krista L. Hyde, McConnell Brain Imaging Center, Montreal Neurological Institute, McGill University, 3801 University Street, Montreal, Quebec, Canada H3A 2B4. E-mail: krista.hyde@mail.mcgill.ca.

D0I:10.1523/JNEUROSCI.3039-07.2007

Copyright $\odot 2007$ Society for Neuroscience $\quad$ 0270-6474/07/2713028-05\$15.00/0
}

recognize familiar tunes (without the aid of lyrics), and are unaware when others (including themselves) sing out of tune. Most notably, amusics fail to detect "wrong notes" (out of key notes) in conventional but unfamiliar melodies (Ayotte et al., 2002). Overall, the behavioral evidence indicates that congenital amusia is caused by a severe deficit in the processing of pitch information, whereas time processing is less affected (Hyde and Peretz, 2004; Foxton et al., 2006). However, much less is known about the neural correlates of this disorder.

Previously, we used voxel-based morphometry (VBM) (Ashburner and Friston, 2000) to investigate structural brain differences between amusics and musically intact controls (Hyde et al., 2006). We found that amusics had reduced white matter in the right inferior frontal gyrus (IFG), and that this anatomical anomaly was related to performance on pitch-based musical tasks. We postulated that the decrease in white matter was indicative of an impoverished white matter communication in a right hemisphere-based network involving the inferior frontal cortex and the right auditory cortex. The decreased white matter in the right IFG of amusics was associated with an increase in gray matter. This inverse relationship between tissue classes may be attributable to the partial volume effect which occurs in brain regions where voxels contain both white and gray matter, or could also reflect a true gray matter increase in the right IFG of amusics. Thus, it was not possible to conclude from these VBM findings alone whether the differences observed truly corresponded to white or gray matter differences.

In the present study, we aimed to specifically investigate the cortical morphometry of the amusic brain by way of cortical thickness measures, a value-added brain morphometric approach to VBM. Cortical thickness provides a specific and direct index of cortical morphology by measuring the distance between 
Table 1. Subject characteristics

\begin{tabular}{lllc}
\hline & Characteristics & Amusics & Controls \\
\hline Montreal & Age in years (SD) & $56.0(10.9)$ & $54.9(6.0)$ \\
& Gender & $13(9 \mathrm{~F} ; 4 \mathrm{M})$ & $19(10 \mathrm{~F} ; 9 \mathrm{M})$ \\
& Education in years (SD) & $16.3(1.7)$ & $16.9(1.8)$ \\
& Global musical score/30 (SD) & $19.5(2.2)$ & $27.0(1.4)$ \\
Newcastle & Age in years (SD) & $54.4(12.6)$ & $54.6(4.6)$ \\
& Gender & $8(4 \mathrm{~F} ; 4 \mathrm{M})$ & $7(4 \mathrm{~F} ; 3 \mathrm{M})$ \\
& Education in years (SD) & $16.8(2.5)$ & $17.2(2.6)$ \\
& Global musical score/30 (SD) & $20.3(1.3)$ & $26.7(1.3)$ \\
\hline
\end{tabular}

F, Female; M, male. Global musical score corresponds to the average score (/30) over all six tests on the Montreal Battery of Evaluation of Amusia.

the white matter surface and the gray matter/cerebral spinal fluid intersection (MacDonald et al., 2000). Thus, unlike VBM, it generates a specific and quantifiable metric of a cortical anatomical property that is biologically meaningful because one can actually measure the thickness in terms of an absolute distance in millimeters. We expected to find cortical thickness differences in the right IFG and also in the right auditory cortex, given its role in musical pitch processing (for review, see Hyde et al., 2006). Although the VBM technique did not uncover any difference in the auditory cortex, we hoped that the cortical thickness technique would reveal such differences because it is a more specific structural brain metric relative to VBM. However, it was not possible to predict whether cortical thickness would decrease or increase in the predicted regions. Thickness decreases might be expected because regional decreases in gray matter are typically correlated with performance deficits in adults with neurodevelopmental disorders, for example in specific language impairment (Watkins et al., 2002). Alternatively, our VBM findings of increased gray matter in the right IFG of the amusic brain suggest that amusics may have regional thicker cortex in the right IFG.

\section{Materials and Methods}

Subjects. There were 47 participants, including 21 amusics and 26 matched controls, who originated from two independent samples. These subjects were the same as those who participated in the VBM study (Hyde et al., 2006). Data were acquired at two collaborating sites, in Montreal (13 amusics and 19 controls) and Newcastle (eight amusics and seven controls), in an effort to maximize the sample size. The controls were musically intact, but had no formal musical training. Amusic subjects were matched to controls in terms of age, gender, education and handedness (Table 1). All amusic subjects had been thoroughly evaluated on previous testing sessions (Ayotte et al., 2002; Foxton et al., 2006), and identical criteria were used for subject selection in both samples. All amusics had normal intellectual, memory and language skills, but were impaired relative to normal controls on a standardized battery of musical tests used to diagnose congenital amusia, the Montreal Battery of Evaluation of Amusia (MBEA). The MBEA involves six tests, including melodic, rhythmic, metric, and recognition memory tests, that have been described in detail previously (Peretz et al., 2003). The global musical scores on the MBEA (averaged over all six tests) served to relate brain anatomy, in terms of cortical thickness, to performance. The present research was approved by the ethics committees of the Montreal Neurological Institute, of the Institut Universitaire de Gériatrie de Montréal, and of the University of Newcastle-upon-Tyne. Informed consent was obtained from all participants.

Scanning protocol and morphometric analyses. T1-weighted magnetic resonance imaging (MRI) sequences were previously acquired for the 21 amusic and 26 musically intact controls on a Siemens (Erlangen, Germany) Sonata 1.5 Tesla scanner at both sites (in Newcastle and Montreal) according to the same MR acquisition parameters: a three-dimensional fast-field echo scan with 160 slices, $1 \mathrm{~mm}$ thick, a repetition time of $22 \mathrm{~ms}$, an echo time of $9.2 \mathrm{~ms}$, and a flip angle of 30 (Hyde et al., 2006). Data for all subjects were processed in the same way using a cortical thickness

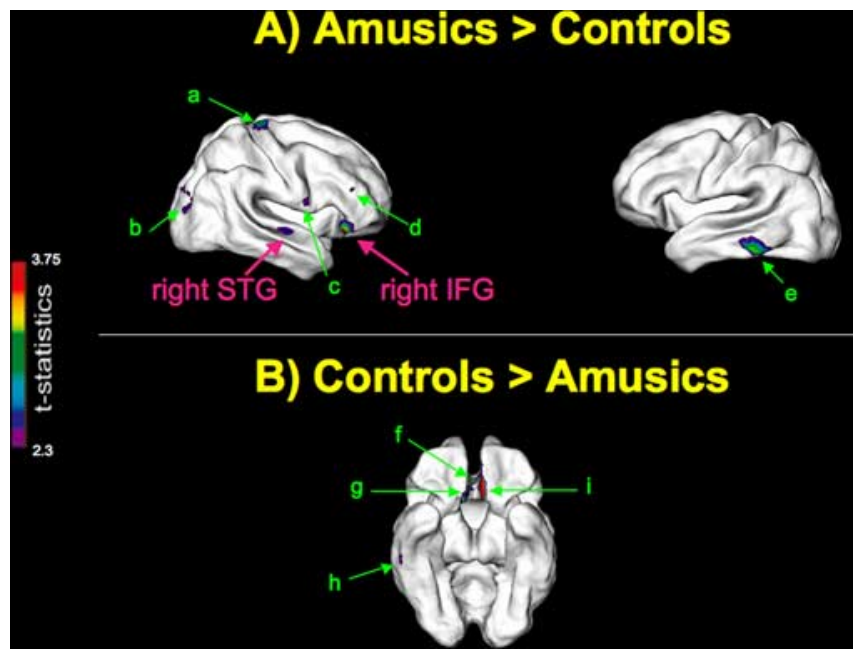

Figure 1. Group cortical thickness differences. Results from the statistical analysis of data from 21 amusics versus 26 controls are displayed at each vertex of the surface of a standardized brain in terms of a tstatistical color map. $\boldsymbol{A}$, Areas of significant thickness increases in the amusic brain relative to controls. $\boldsymbol{B}$, areas of significant thickness decreases in the amusic brain relative to controls. Predicted group cortical thickness differences are in pink, and nonpredicted differences in green as follows: right superior precentral gyrus (a), right lateral occipital gyrus (b), right inferior precentral gyrus (c), right middle frontal gyrus (d), left inferior temporal gyrus (e), right anterior cingulate $(\mathrm{f})$, right medial orbital frontal gyrus $(\mathrm{g})$, right inferior temporal gyrus (h), left medial orbital frontal gyrus (i).

pipeline developed at the Montreal Neurological Institute (Lerch and Evans, 2005). Each T1-weighted image volume was corrected for signal intensity nonuniformity and linearly transformed into standardized stereotaxic space. The transformed images were then classified into gray matter, white matter, and CSF using an automatic tissue classification algorithm. The white and gray matter surfaces were then fitted using deformable spherical mesh models, resulting in two surfaces with 81,920 polygons each. The surface deformation algorithm works by first fitting the white matter surface, then expanding outward to find the gray matter and cerebral spinal fluid intersection. Each vertex (or point) on the white matter surface is closely related to its gray matter surface counterpart and cortical thickness can thus be defined as the distance between linked vertices. Cortical thickness was measured at every vertex and blurred using a $30 \mathrm{~mm}$ surface-based kernel.

Statistical analyses were then performed on the cortical thickness data in $n=47$ subjects. Linear mixed models using the restricted maximum likelihood estimation method were used in the statistical analyses to account for scanning site. Mixed models extend linear models by incorporating random effects, in this case scanner site, which can best be regarded as an additional error term. Results were thresholded at a whole-brain level using the false discovery rate theory (Genovese et al., 2002) with $q=0.05$. This means that only $5 \%$ of the significant results may be false positives, or rather that we are $95 \%$ confident that the significant results are true positives. The results were deemed significant at a $t$ threshold of $2.5(p<0.05)$. There were no significant effects of gender and age on the cortical thickness values.

\section{Results}

\section{Group comparison}

A "whole-brain" group comparison between amusics and controls of mean cortical thickness over the entire brain (averaged over all vertices) did not reveal any significant thickness differences $(t=0.24 ; p=0.81)$. We next performed a "regional" group comparison of cortical thickness at each vertex on the cortex to identify regional cortical thickness differences using the wholebrain statistical threshold specified above. Amusics had significantly thicker cortex relative to controls in the pars orbitalis of the right IFG, in the vicinity of Brodmann areas (BA) 47 and 11 ( $x=$ 


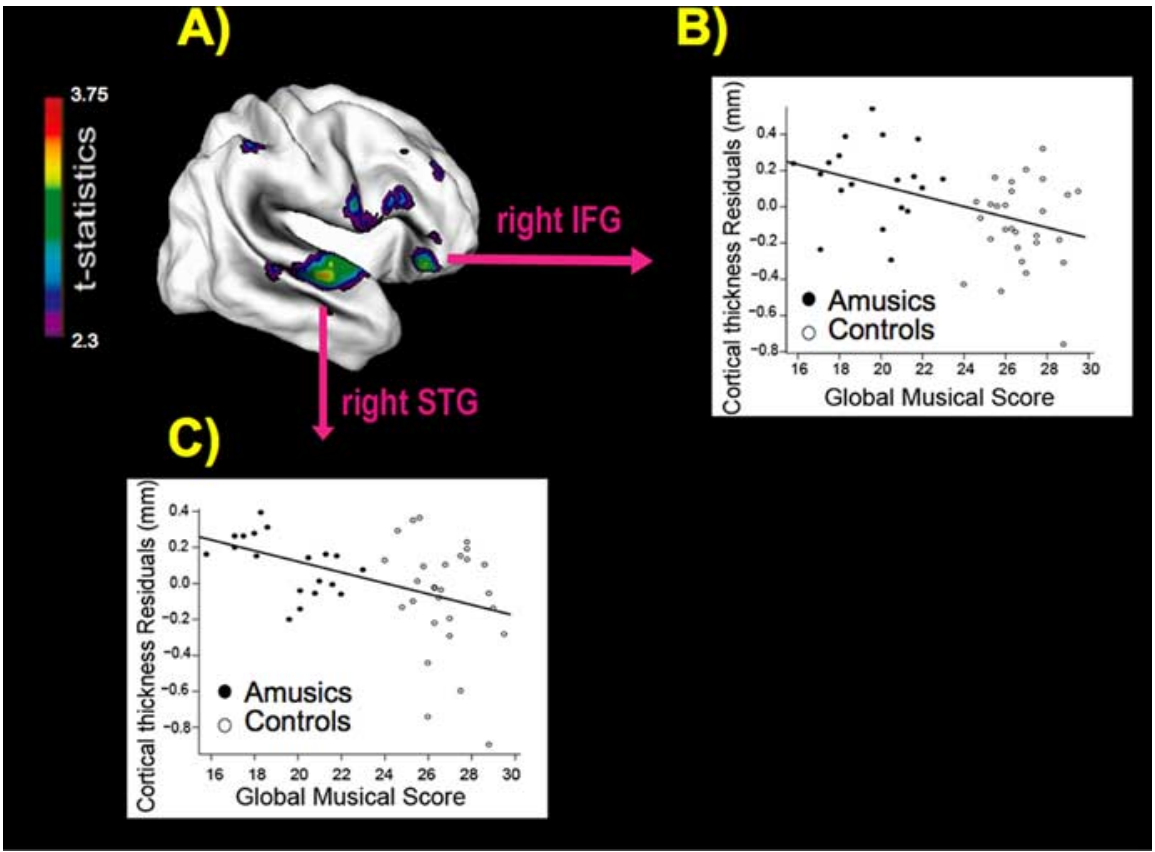

Figure 2. Regression of cortical thickness and global musical scores. $\boldsymbol{A}$, Results from the statistical analysis of data from $n=47$ subjects (21 amusics and 26 controls) are displayed at each vertex of the surface of a standardized brain in terms of a $t$ statistical color map. $\boldsymbol{B}, \boldsymbol{C}$, The regression of cortical thickness residuals and global musical scores at the right IFG is illustrated in $\boldsymbol{B}$, and at the right STG in $\boldsymbol{C}$. These residual plots reflect the cortical thickness values after having factored out the effect of scanning site.

significant negative correlation between cortical thickness and global musical scores in the same left inferior temporal gyrus $(t=-3.1)$ as found in the group comparison. Significant positive correlations, where cortical thickness increased with increasing performance scores on the musical tests, were found in left $(t=4.9)$ and right $(t=3.1)$ medial orbital frontal gyri, right anterior cingulate $(t=3.3)$, and right inferior temporal gyrus $(t=2.7)$, as found in the group comparison.

Given that the subtests of the MBEA measure various aspects of music perception, we also performed regression analyses of cortical thickness with the scores on the individual subtests. The results from the correlational analysis with the global MBEA measure were consistent across individual subtests, and no one particular subtest was more highly correlated with anatomical differences relative to another. Thus, we focus our discussion on the brain-behavioral correlational analyses with the global measure of the MBEA, which is currently the diagnostic criterion for congenital amusia.

\section{Discussion}

$45, y=28, z=-11 ; t=3.8$ ) (Fig. $1 A$ ). Amusics also had significantly thicker cortex relative to controls in a right secondary auditory area on the superior temporal gyrus (STG), in the vicinity of BA $22(x=60, y=-12, z=5 ; t=2.6)$ (Fig. $1 A)$. Regional cortical thickness differences were found in other brain areas that were not predicted a priori. We found significant thickness increases in the amusic brain relative to controls in right frontal regions, including middle frontal $(t=2.5)$, superior $(t=3.2)$, and inferior $(t=2.6)$ right precentral gyrus, as well as in the right putamen $(t=3.1)$, right lateral occipital gyrus $(t=2.5)$, and in the left inferior temporal gyrus $(t=3.3)$ (Fig. 1A). Significant decreases in thickness in the amusic brain relative to controls were found in left $(t=-4.9)$ and right $(t=-3.4)$ medial orbital frontal gyri, right anterior cingulate $(t=-3.7)$ and right inferior temporal gyrus $(t=-2.7)$ (Fig. $1 B)$.

\section{Regression analyses with performance on musical tests}

We performed a regression of cortical thickness with the global musical battery scores (averaged over the six tests of the MBEA) at each vertex, across all subjects, to detect brain regions where cortical thickness correlates with performance on the musical tests. The regression analyses revealed a negative correlation between cortical thickness and global musical scores in almost the identical right IFG region $(x=45, y=30, z=-13$; $t=-3.3)$ and right secondary auditory region $(x=60, y=-10, z=6$; $t=$ -3.3 ) (Fig. $2 A$ ) as found in the group analysis. To illustrate this relationship between brain morphometry and behavior, we plotted the cortical thickness obtained at both of these areas for each subject as a function of their global musical scores on the MBEA. Cortical thickness decreased with increasing performance scores on the musical tests, at both the right IFG peak $(r=0.45 ; p=$ $0.002)$ (Fig. $2 B)$ and the right secondary auditory peak $(r=0.45$; $p=0.002)($ Fig. $2 B)$.

The regression analyses also revealed significant correlations in other brain areas that were not predicted a priori. We found a
As predicted, here we found brain morphological anomalies in terms of cortical thickness in the right IFG and the right auditory cortex of amusic subjects. The involvement of the right IFG in the vicinity of BA 47 and $11(x=45, y=28, z=-11)$ is consistent with the localization of the neural anomaly found with the VBM technique ( $x=39, y=42, z=-3$ ) (Hyde et al., 2006). The present findings are novel in that the cortical thickness technique allows us to conclude that there exists a cortical anomaly. This anomaly is likely related to a cortical malformation in development that in turn affects white-matter connectivity as will be discussed below. Moreover, the cortical thickness technique proved to be more sensitive than VBM in revealing an expected neural anomaly in the right auditory cortex of amusics.

\section{Congenital amusia related to a structural neural anomaly along a right frontotemporal pathway}

The correlation found between cortical thickness and the global musical scores at both the right IFG and right auditory area is consistent with the functional literature demonstrating that these regions are important in musical pitch processing. These regions have been implicated in the active retention of pitch in melodies (Zatorre et al., 1994), and in the processing of tonality in the detection of unexpected chords in a harmonic context (Tillmann et al., 2003). Brain-lesion studies have shown that the right auditory cortex is critical in melodic perception (Peretz, 1990), and the perception of pitch direction (Johnsrude et al., 2000). Neuroimaging studies have shown that the right auditory cortex is key in sequential pitch processing (Zatorre et al., 1994; Patterson et al., 2002), and in fine-grained pitch discrimination (Hyde et al., 2007), which in turn is thought to underlie the musical disturbance in amusia (Hyde et al., 2004).

The right inferior frontal cortex is connected to the auditory cortex by frontotemporal fiber tracts (Hackett et al., 1999) that seem to be less developed in the amusic brain. In our VBM study 
(Hyde et al., 2006), we proposed that the white matter changes observed in the right IFG may reflect an anomalous connectivity between auditory and frontal cortical areas that are critical to the development of tonal perceptual competence. The fact that cortical thickness differences were found in both the right IFG and right auditory cortex supports the idea that structural neural anomalies along a right frontotemporal pathway may contribute to congenital amusia.

\section{How specific is congenital amusia to the musical domain?}

The finding of cortical thickness differences in areas outside the predicted musically relevant regions of the right inferior frontal gyrus and auditory cortex, raises the question as to whether amusic individuals may have deficits in domains other than music. It would be difficult at this stage to interpret all of the nonpredicted differences because these regions subserve multiple functions and the functional consequences of thickness anomalies uncovered in these locations remain to be identified. However, these results may signal that there are aspects of congenital amusia that remain to be explored. For example, it has been suggested previously that congenital amusia may be associated with deficits in spatial processing (Douglas and Bilkey, 2007). Thus, the nature of the disorders associated with the condition of congenital amusia remains an important question to be studied further. We focus the remainder of our discussion on the brain regions where we expected to find differences, namely the right IFG and auditory cortex.

\section{Congenital amusia related to a malformation in cortical development?}

The thicker cortex observed in the right IFG and right secondary auditory area of the amusic brain may be related to a malformation in cortical development, lack of normal neuronal pruning during development, or an environmental influence on brain structure in terms of lack of same amount of musical exposure in amusics relative to musically intact controls. Although the last two possibilities remain open, we argue in favor of the cortical malformation hypothesis on the basis of compelling brain histological findings revealing cortical malformations in other neurological conditions. Histological findings in dyslexia (Galaburda et al., 1985) and various forms of epilepsy (Guerrini et al., 2003) are informative regarding the type of developmental cortical malformation that can give rise to increases in cortical thickness. In the normal developing brain, neurons generated deep in the brain follow a highly specific migratory pathway to navigate to predetermined locations that define the six functional layers of the adult cerebral cortex. However, in some individuals, subsets of neurons fail to migrate into the developing cerebral cortex, and remain as nodules of neurons that line the deep brain or ventricular surface (heterotopic nodules), which, in turn, may lead to increased cortical thickness (Guerrini et al., 2003; Guerrini and Marini 2006). This form of abnormal migration, called periventricular heterotopia, typically manifests clinically as epilepsy (Guerrini et al., 2003), and has been reported previously to be associated with dyslexia (Chang et al., 2005). Abnormal neuronal migration can also lead to abnormal structural cortical organization, for example in the form of polymicrogyria, which is characterized by an excessive number of small and prominent convolutions spaced out by shallow and enlarged sulci (Guerrini and Marini, 2006). This abnormal packing of microgyri may also lead to increased cortical thickness (Guerrini and Marini, 2006), and results in various clinical manifestations, including dyslexia (Galaburda et al., 1985) and epilepsy (Guerrini et al., 2003). To- gether, the histological evidence of malformations in cortical development that underlie regional cortical thickness found in dyslexia and epilepsy, suggests that similarly, congenital amusia may be related to abnormal neural migration.

\section{Malformations in cortical development disturbs brain connectivity}

The question is then how to relate the VBM finding of increased gray matter in the right IFG of amusics to the white matter decrease observed in the same area. This discrepancy may be resolved by evidence from human (Chang et al., 2005) and animal (Rosen et al., 2000) studies that indicate that cortical malformations in development may lead to disruptions in white matter connectivity in proximal brain regions. This may occur as a result of heterotopic nodules forming white matter connections with each other and with overlying cortex, thereby disturbing local brain connectivity (Kakita et al., 2002). Support for this idea comes from a previous study of individuals with periventricular heterotopia that were also shown to have difficulties in reading processing speed (Chang et al., 2005). The authors proposed that the heterotopic nodules present diffusely along the ventricles in these individuals could account for their reading impairments by interfering with the white matter tracts critical for reading processing speed. Additional support comes from an animal model in which rats that were induced prenatally with cortical malformations in the form of microgyria showed reduced connectivity between neighboring brain regions (Rosen et al., 2000).

In sum, cortical malformations can interfere with white matter connectivity. This, in turn, may account for the fact that we observed in our VBM study (Hyde et al., 2006) both a decrease in white matter and an increase in gray matter in the right IFG of amusics. Therefore, the results are compatible with the view that normal musical competence may depend on the structural integrity of a right frontotemporal pathway, involving the right inferior frontal and auditory cortex (Hyde et al., 2006).

\section{Could the anomalous right frontotemporal pathway be related to a genetic component?}

Although the genetics of congenital amusia are presently unknown, it is likely that this condition is related to a genetically based cortical malformation. Support for this claim comes from evidence of a genetic basis for various neurological disorders known to be related to cortical malformations, such as dyslexia (Smith et al., 1998), and specific language impairment (Lai et al., 2001). Additional support for this idea comes from a twin study that demonstrated that musical pitch encoding, which is critically impaired in all amusics cases tested in our laboratory to date, may have a heritable component (Drayna et al., 2001). Moreover, about half of the first-degree relatives of the amusic cases are also musically impaired (Peretz et al., 2007). Thus, the present findings of cortical thickness differences in the amusic brain may serve as a neurogenetic marker of congenital amusia and provide the possibility to distinguish between the brains of afflicted and nonafflicted individuals.

\section{Conclusion}

In conclusion, we conceive the thicker cortex observed here in the right IFG and right auditory cortex of amusic individuals relative to controls, as related to a cortical malformation. In turn, a cortical malformation may affect white-matter connectivity in proximal brain regions. Thus, a disturbed frontotemporal network involving cortical regions known to be implicated in musical processing, namely the right inferior frontal and auditory cortex, 
appears to be related to the musical impairments in congenital amusia. These findings have implications for the understanding of normal acquisition of musical abilities. Moreover, these results motivate further studies on the neural correlates of congenital amusia. Future neuroanatomical studies using postmortem microstructural histology and receptor methods need to be performed to characterize more precisely any cortical malformations in the amusic brain.

\section{References}

Ashburner J, Friston KJ (2000) Voxel-based morphometry-the methods. NeuroImage 11:805-821.

Ayotte J, Peretz I, Hyde K (2002) Congenital amusia: a group study of adults afflicted with a music-specific disorder. Brain 125:238-251.

Chang BS, Ly J, Appignani B, Bodell A, Apse KA, Ravenscroft RS, Sheen VL, Doherty MJ, Hackney DB, O'Connor M, Galaburda AM, Walsh CA (2005) Reading impairment in the neuronal migration disorder of periventricular nodular heterotopia. Neurology 64:799-803.

Douglas KM, Bilkey DK (2007) Amusia is associated with deficits in spatial processing. Nat Neurosci 10:915-921.

Drayna D, Manichaikul A, de Lange M, Snieder H, Spector T (2001) Genetic correlates of musical pitch recognition in humans. Science 291:1969-1972.

Foxton JM, Nandy RK, Griffiths TD (2006) Rhythm deficits in "tone deafness." Brain Cogn 62:24-29.

Galaburda AM, Sherman GF, Rosen GD, Aboitiz F, Geschwind N (1985) Developmental dyslexia: four consecutive patients with cortical anomalies. Ann Neurol 18:222-233.

Genovese CR, Lazar NA, Nichols T (2002) Thresholding of statistical maps in functional neuroimaging using the false discovery rate. NeuroImage 15:870-878.

Guerrini R, Marini C (2006) Genetic malformations of cortical development. Exp Brain Res 173:322-333.

Guerrini R, Sicca F, Parmeggiani L (2003) Epilepsy and malformations of the cerebral cortex. Epileptic Disord 5 [Suppl 2]:S9-S26.

Hackett TA, Stepniewska I, Kaas JH (1999) Prefrontal connections of the parabelt auditory cortex in macaque monkeys. Brain Res 817:45-58.

Hyde KL, Peretz I (2004) Brains that are out of tune but in time. Psychol Sci 15:356-360.

Hyde KL, Zatorre RJ, Griffiths TD, Lerch JP, Peretz I (2006) Morphometry of the amusic brain: a two-site study. Brain 129:2562-2570.
Hyde KL, Peretz I, Zatorre RJ (2007) Evidence for the role of the right auditory cortex in fine pitch resolution. Neuropsychologia, in press.

Johnsrude IS, Penhune VB, Zatorre RJ (2000) Functional specificity in the right human auditory cortex for perceiving pitch direction. Brain $123(\mathrm{Pt}$ 1):155-163.

Kakita A, Hayashi S, Moro F, Guerrini R, Ozawa T, Ono K, Kameyama S, Walsh CA, Takahashi H (2002) Bilateral periventricular nodular heterotopia due to filamin 1 gene mutation: widespread glomeruloid microvascular anomaly and dysplastic cytoarchitecture in the cerebral cortex. Acta Neuropathol (Berl) 104:649-657.

Kalmus H, Fry DB (1980) On tune deafness (dysmelodia): frequency, development, genetics and musical background. Ann Hum Genet 43:369-382.

Lai CS, Fisher SE, Hurst JA, Vargha-Khadem F, Monaco AP (2001) A forkhead-domain gene is mutated in a severe speech and language disorder. Nature 413:519-523.

Lerch JP, Evans AC (2005) Cortical thickness analysis examined through power analysis and a population simulation. NeuroImage 24:163-173.

Patterson RD, Uppenkamp S, Johnsrude IS, Griffiths TD (2002) The processing of temporal pitch and melody information in auditory cortex Neuron 36:767-776.

MacDonald D, Kabani N, Avis D, Evans AC (2000) Automated 3-D extraction of inner and outer surfaces of cerebral cortex from MRI. NeuroImage $12: 340-356$.

Peretz I (1990) Processing of local and global musical information by unilateral brain-damaged patients. Brain 113:1185-1205.

Peretz I, Cummings S, Dubé MP (2007) The genetics of congenital amusia (or tone-deafness): a family aggregation study. Am J Hum Genet 81:582-588.

Peretz I, Champod AS, Hyde K (2003) Varieties of musical disorders. The Montreal battery of evaluation of amusia. Ann NY Acad Sci 999:58-75.

Rosen GD, Burstein D, Galaburda AM (2000) Changes in efferent and afferent connectivity in rats with induced cerebrocortical microgyria. J Comp Neurol 418:423-440.

Smith SD, Kelley PM, Brower AM (1998) Molecular approaches to the genetic analysis of specific reading disability. Hum Biol 70:239-256.

Tillmann B, Janata P, Bharucha JJ (2003) Activation of the inferior frontal cortex in musical priming. Brain Res Cogn Brain Res 16:145-161.

Watkins KE, Vargha-Khadem F, Ashburner J, Passingham RE, Connelly A, Friston KJ, Frackowiak RS, Mishkin M, Gadian DG (2002) MRI analysis of an inherited speech and language disorder: structural brain abnormalities. Brain 125:465-478.

Zatorre RJ, Evans AC, Meyer E (1994) Neural mechanisms underlying melodic perception and memory for pitch. J Neurosci 14:1908-1919. 